\title{
PLANAR PHOSPHOLIPID MEMBRANES IN MICROFLUIDICS ENABLING KINETICS STUDIES OF CERAMIDE CHANNELS
}

\author{
Chenren Shao ${ }^{1 *}$, Marco Colombini ${ }^{2}$, and Don. L. DeVoe ${ }^{2}$ \\ ${ }^{1}$ Department of Mechanical Engineering, University of Maryland, College Park, Maryland, USA \\ ${ }^{2}$ Department of Biology, University of Maryland, College Park, Maryland, USA
}

\begin{abstract}
Here we present recent developments on the integration of planar phospholipids membrane (PPM) into microfluidic platform. With robust PPM formed in our polycarbonated based microchip, we can further incorporate one of the most interesting naturally occurring lipids channel ceramide ( $N$-acylated spingosine), and then integrate perfusion pathway to change the reagent condition of aqueous solution in one of the chambers. It demonstrates the ability to study the dynamic responses of ion channel to different perfusion conditions and to capture the kinetic activities which would provide mechanistic insight for ion channels study.
\end{abstract}

\section{INTRODUCTION}

Ceramide ( $\mathrm{N}$-acylated spingosine), a sphingolipid found in mitochondrion membranes, is involved in several important cellular activities, including differentiation, growth suppression, cell senescence and apoptosis. Recent research shows that it may regulate apoptosis by forming stable channels to release mitochondrial proapoptotic intermembrane proteins to the cytoplasm [1-3]. Further understanding of the structure and function of lipid ion channels will require kinetic interaction studies with different ligands such as trehalose and Bcl-2 family proteins [3]. However, traditional in vitro electrophysiology platforms employ large fluid volumes which make it impossible to rapidly and dynamically modulate the local (bio)chemical environment at the channel site. Miniaturization is an obvious solution but simple, cost effective and user friendly microsystems for dynamic ion channel studies remain elusive due to innate fragility of lipid membranes and also from complications associated with fluid controls and electrical access. Previously we reported a convenient fabrication process for a robust microfluidic planar phospholipid membrane (PPM) platform enabling stable membrane formation and ion channel introduction [4]. Here we present recent developments on the integration of perfusion pathways, and demonstrate the ability to capture the kinetic activities of ceramide channels under different dynamic reagent perfusion conditions.

\section{FABRICATION AND MATERIALS}

As shown in Fig. 1, the polycarbonate (PC) microfluidic PPM chip consists of two microchannels, each in a different PC substrate, separated by a $13 \mu \mathrm{m}$ thick polyvinylidene chloride (PVDC) film. The chip-to-world fluidic connection is realized by micro unions and the electrodes are held by nanoports, both from Upchurch, MA. An aperture of $80 \sim 150 \mu \mathrm{m}$ in diameter is fabricated by a precisely controlled hot needle (Fig. 2) as a membrane hosting site. The inset in Fig.1 shows a top view from open well of a PPM site in PVDC film.

The reagents of interest are delivered by syringe pumps through the perfusion channels to bottom chamber, and to waste reservoir located at the end of bottom chamber. In this study, $\mathrm{LaCl}_{3}$, ethylenediaminetetraacetic acid (EDTA) and trehalose are investigated.

Unlike protein ion channels, the highly hydrophobic nature of the lipid ceramide molecule imposes unique challenges for ion channel integration due to their poor solubility. Here we describe a new approach based on pre-mixing ceramide and membrane lipid and using a diffusion "painting" method to encourage efficient and repeatable channel formation. Briefly, a mixture of 1,2-dipalmitoyl-sn-glycero-3-phosphocholine (DPPC), asolectin, and cholesterol (Avanti Polar Lipids, AL) is dissolved in hexane as a pre-coating solution. The solution is pipetted directly onto the PPM site through the open reservoir, and residual hexane is evaporated by a gentle stream of nitrogen. After filling the open reservoir and microchannels with buffer, a glass tip is rolled in a painting solution and gently wiped across the hole. The painting solution has same lipid concentration as pre-coating except has $\mathrm{N}$-hexadecyl-D-erythro-sphingosine $\left(\mathrm{C}_{16}\right.$-ceramide $)$ at 1:50 molar fraction with respect to phospholipids in hexanol/hexadecane (10:1, $\mathrm{v} / \mathrm{v})$. By diffusing hexanol to surrounding buffer, the lipid plug can thin into bilayer and ceramide can eventually assemble into a channel.
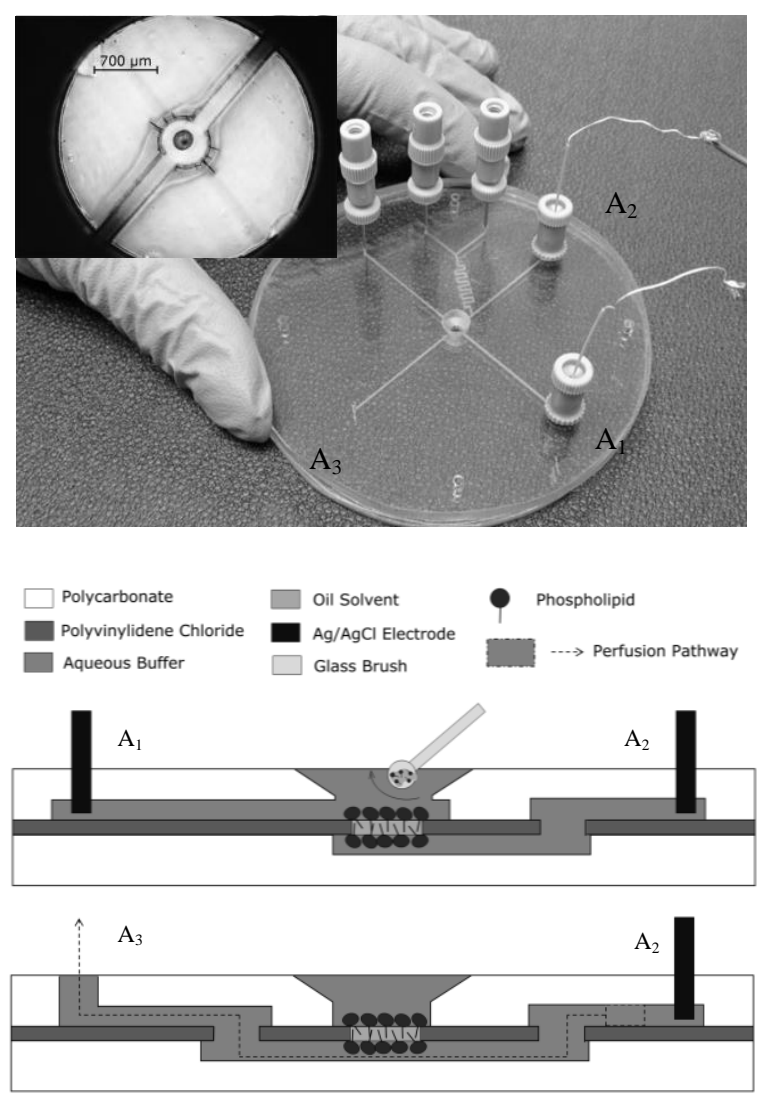

Figure 1: Schematic view of microfluidic PPM chip. Cross section view of $A_{1}-A_{2}$ and $A_{3}-A_{2}$ show the electrical pathway and perfusion pathway respectively. 


\section{EXPERIMENTS AND DISCUSSION}

Unlike previous efforts to develop automated ion channel analysis systems using microfluidics, our goal here is to develop a simple and practical benchtop platform suitable for biophysical investigation of ion channels, including both protein and lipid channels, requiring the application of time-varying chemical concentrations on either side of the channel membrane. This is especially useful to study the physiological functions of relevant ion channels such as ceramide.

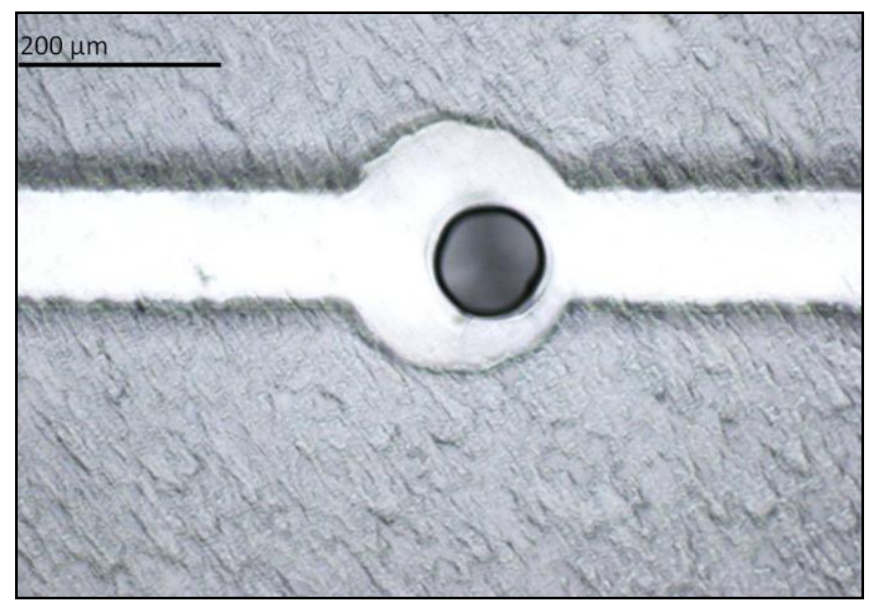

Figure 2: A 100X view of an aperture burnt by a hot needle on $P V D C$ film right above bottom channel. The diameter of the aperture ranges from $80 \mu \mathrm{m}$ to $150 \mu \mathrm{m}$ in different chips.

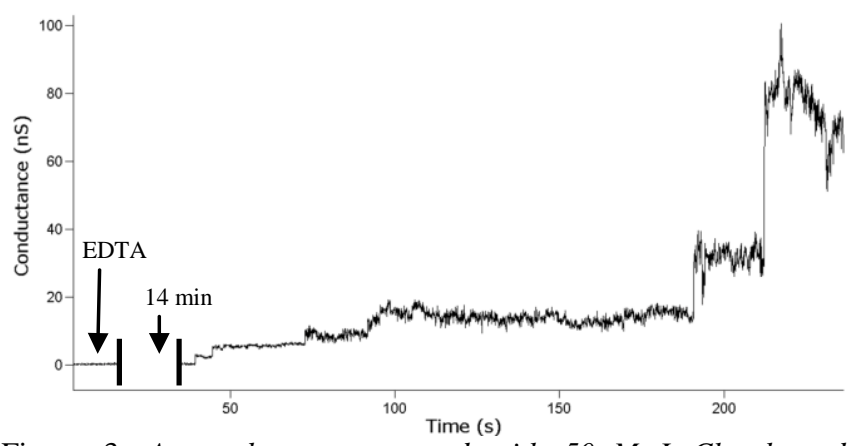

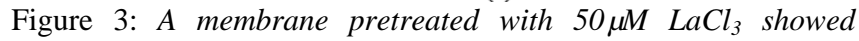
ceramide formation activities when 50 $\mu M$ EDTA is injected through bottom channel. Buffer contains $1 \mathrm{M} \mathrm{KCl}, 1 \mathrm{mM} \mathrm{MgCl}_{2}, 20 \mathrm{mM}$ PIPES, pH 6.98 .

An example of this capability using $\mathrm{LaCl}_{3}$ and EDTA is revealed in Fig. 3. $\mathrm{LaCl}_{3}$ is known to dissemble ceramide channels, while EDTA works as chelator, deactivating the inhibiting function of $\mathrm{La}^{3+}$ ions. Upon the introduction of $50 \mu \mathrm{M} \mathrm{La}^{3+}$ through the lower mirochannel, ceramide channel formation is inhibited. Next, $50 \mu \mathrm{M}$ EDTA is controllably delivered to the membrane site with flow rate $0.2 \mu \mathrm{L} / \mathrm{min}$. Progressive chelation of $\mathrm{La}^{3+}$ by EDTA ultimately leads to formation of a ceramide channel, with a maximum conductance up to $80 \mathrm{nS}$. The total waiting time for the formation is 14 minutes, matching with the time needed by EDTA frontier to be pumped to the bottom chamber.
In another example, trehalose, a sugar with a specific arrangement of hydroxyls, is predicted to interact with hydroxyl groups in the inner wall of a ceramide channel and alter the pore size. In a preliminary test shown in Fig.4, we successfully see timely response of ceramide channel upon addition and removal of trehalose.

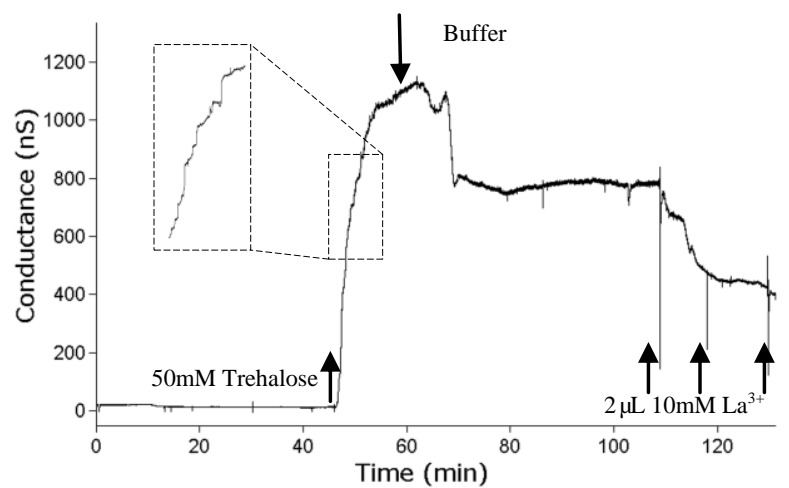

Figure 4: A two-hour current recording of ceramide interacting with trehalose. The perfusion of trehalose enhanced the conductance from $\sim 20 n S$ to $\sim 1150 n S$. Removal of trehalose by buffer decreased it to $\sim 800 \mathrm{nS}$. Following addition of $\mathrm{La}^{3+}$ reduce the conductance further. Buffer contains $0.25 \mathrm{M} \mathrm{KCl}, 0.25 \mathrm{mM}$ $\mathrm{MgCl}_{2}, 20 \mathrm{mM}$ PIPES, pH 6.98 .

\section{REFERENCES}

[1] L.J. Siskind and M. Colombini, "The Lipids $\mathrm{C}_{2^{-}}$and C16-ceramide Form Large Stable Channels: Implications for Apoptosis", Journal of Biological Chemistry, 275, 38640 (2000).

[2] L.J. Siskind, A. Davoody, N. Lewin, S. Marshall and M. Colombini, "Enlargement and Contracture of C2-Ceramide Channels." Biophysical Journal Biophysical Journal, 85, 1560 (2003).

[3] L. J. Siskind, "Mitochondrial Ceramide and the Induction of Apoptosis." Journal of Bioenergetics and Biomembranes. 37, 143 (2002).

[4] C. Shao, M. Colombini, D. L. DeVoe, "Planar Phospholipid Membrane Formation in Open Well Thermoplastic Chips". 2009 ASME International Mechanical Engineering Congress and Exposition, Lake Buena Vista, FL, 11/13-19/09.

\section{CONTACT}

*Chenren Shao, tel: +1-301-405-1023; chenren@umd.edu 